INTERNATIONAL DESIGN CONFERENCE - DESIGN 2018

https://doi.org/10.21278/idc.2018.0547

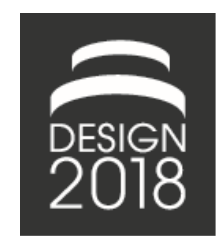

\title{
EXPLORATION OF COGNITIVE DESIGN BEHAVIOUR DURING DESIGN CRITIQUES
}

\author{
J. Milovanovic and J. S. Gero
}

\begin{abstract}
The design studio pedagogy fosters a learning by designing model for students to develop designing skills. A methodology, based on protocol analysis and the FBS framework, is proposed to measure actors' design cognitive behavior and interaction throughout design critiques. Those metric measurements provide a quantitative understanding of their design activity during the critiques. A case study of four architectural critiques of a team of two students, across a semester, illustrates our methodology. The results point out differences in each actor's behavior, and their evolution across sessions.
\end{abstract}

Keywords: design education, design cognition, protocol analysis

\section{Introduction}

The design studio format as a form of educational pedagogy fosters a learning by doing environment for students to learn how to design by doing designing (Schön, 1985; Dutton, 1987; Dinham, 1989). Design critiques are milestones during the design studio process where students get feedback and guidance from their tutors to proceed with their design activity (Anthony, 1991; Oh et al., 2013). During designing the constructiveness of the design space pushes the designer to frame and reframe the problem space along with the generation of the design solution (Schön, 1988). This process is expressed by a coevolution of the problem and the solution spaces that make up the design space (Maher and Poon, 1996; Dorst and Cross, 2001). The navigation of a design space is described by processes that transition from the problem space to the solution space, and from the solution space to the problem space. These transitions are the core of the design activity and are synthesized in the literature as analysis, synthesis and evaluation (Asimov, 1962; Lawson, 2006). Various models are adapted from that general framework, for example, seeing, framing, moving and reflecting (Schön, 1985); generation, transformation, evaluation (Visser, 2006); or the eight design process from the FBS ontology (Gero, 1990; Gero and Kannengiesser, 2007). In this paper, we explore the nature of the design activity during critique sessions that involve the interactions of the critique participants (tutors and students).

The design studio, as a form of pedagogy, is discussed in the literature through many lenses, which provides rich descriptions of its framework. However, there is a lack of metric measurements of its actors' cognitive activities to provide a quantitative understanding of how actors behave during design critiques. Defining methods to measure the design activity during the critique is a way to evaluate the different studio pedagogies by comparing their measurements (how students and tutors interact and design). The aim of the paper is to explore different methodological tools to analyze and describe design studio critiques and to formulate hypotheses to be tested in future studies. Each tool is illustrated by a case study of four architectural design critique sessions for the same team of two students. Each session was analyzed using the protocol analysis method (Ericsson and Simon, 1984; Gero and McNeill, 1998; Kan and Gero, 2017), using two coding schemes: the theory-based design process coding scheme based 
on the FBS ontology (Gero, 1990; Gero and Kannengiesser, 2007) and an interaction coding scheme to express actors' interactions during the critique's conversation. To represent and study the design activity during the critique, we used multiple tools to analyze the coded protocols: general statistical distribution of the coded segments, a refined version of the P-S index (Jiang et al., 2014) of each session, a first order Markov model and correspondence analysis between our two coding schemes.

\section{Design critiques}

\subsection{Critique setting}

\subsubsection{Activities in the critique}

Design critiques set an environment for students to get feedback on their design. A typical sequence in a design critique session is as follows: students start to present their work; tutors often listen and observe; they critique students' designs by evaluating their project, reframing the design problem space and addressing those problems. Questioning, analyzing, reflecting and proposing possible solutions or formalizing guidelines are common actions throughout the critiques (Schön, 1985; Oh et al., 2013). Those actions can be referred to as acts of designing, where talking and sketching intertwine. In The Design Studio, Schön (1985) identifies four critique action types performed by the instructor and the student that function in pairs: telling (instructor) and listening (student); and demonstrating (instructor) and imitating (student). The first set corresponds to explicit formulation of design knowledge, like specific instructions to follow, theoretical solutions, questions about representations' format or references to precedents; and the second refers to a situation where designing occurs through the tutor's demonstration, that includes framing and reframing, analyzing and proposing changes (Schön, 1985; Goldschmidt et al., 2010). Studio specific activities, in relation to learning, were classified by Heylighen et al. (1999) in four distinct moments: demonstrations employing both verbal and graphic design language, descriptions of a design principle, reference to precedent, and the teacher's own designing and sketching. We recognize two main activities, the first in relation to designing or reflection-in-action and the second that appears as a reflection-on-action, based on an analysis of the process correlated with the discussion of methods, general theories or precedents to support and foster students' designs.

\subsubsection{Actors in the critique}

Tutors in the design studio are often experienced designers who serve as role models for their students. Schön (1985) describes the teacher as a coach or facilitator, while Goldschmidt et al. (2010) further developed definitions of the teacher's profile (source of expertise, coach, 'buddy') by analyzing the critique sessions of three different teachers, identifying diverse facets of teacher performance and their capacity to adapt their remarks depending on students' reactions to the critique. Schön's (1985) definition of critique activities, places students in an observant position of learning by imitation. For Webster (2008) and Mewburn (2012), this vision of the student/teacher interaction is questionable. A student-centered learning approach implies that the student must be active in order to enhance their learning outcome.

\subsection{Studies on analyzing design critiques}

Protocol analyses of design critiques or reviews are not as well developed as studies of designing. Although recently, the publications of articles from the Design Thinking Research Symposium 10, which focused on design reviews and critiques, extended the body of research on this topic (Adams et al., 2016a, 2016c; Adams and Siddiqui, 2016). The frameworks chosen to explore design critiques vary pointing out different aspects of design critiques concerning designing, coaching, reasoning and knowledge building. The study of critique conversation, showing actors' cognitive behavior or emotional reactions, reveals the presence of design and co-design activities in review sessions (Dorta et al., 2016; Ferreira et al., 2016; Gero and Jiang, 2016) and divergence in their construct of design cognitive actions (Khaidzir and Lawson, 2013). Diverse sequences of reasoning and switches between knowledge organization have shown to enhance students' design quality (Wolmarans, 2016) and improve their learning outcome (Cardoso et al., 2016; Dong et al., 2016). The relevance of critiques' activities mainly relies on the 
capacity of tutors to clarify their expectations (Dinham, 1989), to exploit appropriate coaches' strategies (Goldschmidt, 2002; Goldschmidt et al., 2010; Adams et al., 2016b) and to choreograph crit sessions (for the remainder of the paper we will use the shorthand "crit" to refer to critique) to provide an enriching environment for students to develop their skills (Mewburn, 2012; Oak and Lloyd, 2016).

\section{Methodology}

\subsection{Protocol analysis}

To explore the design activity during the design critiques, the protocol analysis method was used with two different coding schemes. The protocol analysis method aims to analyze cognitive process during a design activity, by transcribing and encoding think-aloud protocols or design conversations (Ericsson and Simon, 1984; Gero and McNeill, 1998; Kan and Gero, 2017). This method can be adapted to study the design critique discussions. The conversation transcript is segmented in units, depending on the content of the verbal data. Each segment is associated with a code. Using the same method for each design review session allows comparative analysis between the sessions.

We focused on the design activity during each session and how each actor influences that activity. For that reason, the first coding scheme is constituted of the eight theoretical design process described by the FBS ontology (Gero, 1990; Gero and Kannengiesser, 2007; Kan and Gero, 2017) and the second scheme, called interaction, represents the interactions between tutors and students.

\subsection{FBS ontology, design process and actor's interactions}

The FBS ontology proposes a theoretical description of design activities, represented by design issues (Function, Behavior and Structure) and eight different design process, which represent a transition from one design issue to another one (Figure 1). The function (F) of a designed object is defined as its teleology or what the object is for. The behavior (B) of that object is either derived from the structure (Bs) or expected $(\mathrm{Be})$, which is the behavior of the object in the designer's head, associated with its Function. The structure (S) represents the components of an object and their compositional relationships. Requirements (R) come from outside the designer. They could be the clients' requirements for example, but also legislative requirements. Design descriptions (D) are the documents of the design object like plans, models or graphs.

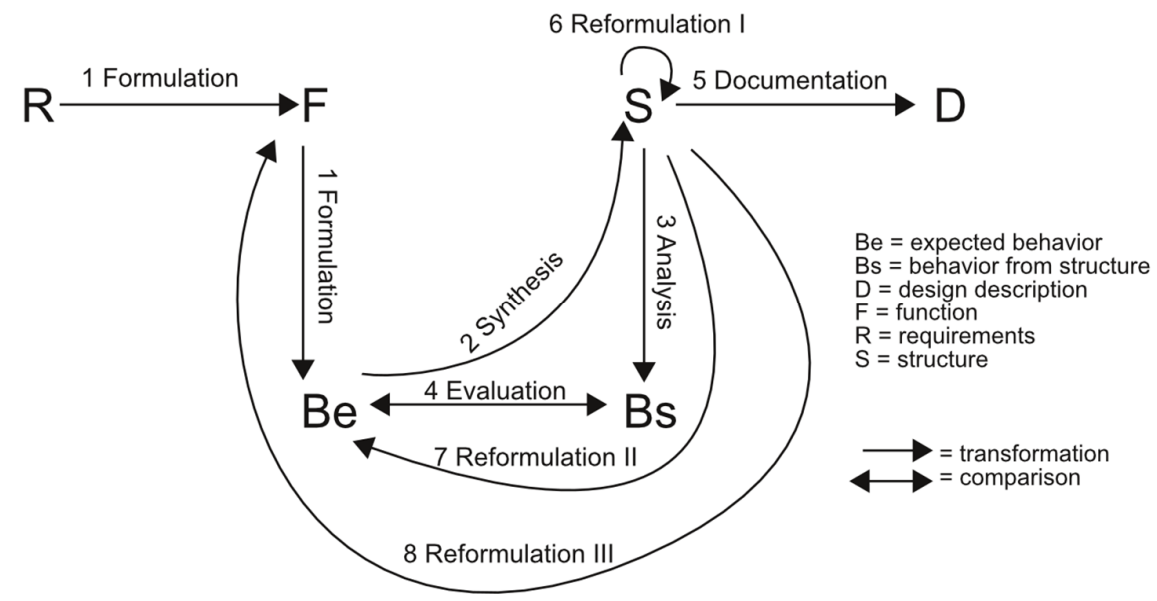

Figure 1. FBS ontology showing the design issues and the design process (Gero, 1990; Gero and Kannengiesser, 2004)

Eight theoretical design process are described by the FBS ontology: Formulation is a transformation of requirements $(\mathrm{R})$ into function $(\mathrm{F})$ or function $(\mathrm{F})$ into expected behavior $(\mathrm{Be})$; Synthesis is a transformation of an expected behavior (Be) into a solution producing structure (S); Analysis is an expression of a behavior (Bs) from a structure (S) design issue; Evaluation is a comparison between a behavior derived from structure (Bs) and an expected behavior (Be), or vice versa; Documentation is the production of a representation (D) from a structure (S) design issue; Reformulation 1 is a transition from 
a structure (S) issue to another structure ( $\mathrm{S}$ ) issue; Reformulation 2 is a transition from a structure (S) issue to an expected behavior $(\mathrm{Be})$ and Reformulation 3 is a transition from a structure $(\mathrm{S})$ issue to function $(\mathrm{F})$. During design critiques, there are at least two types of actors: students $(\mathrm{S})$ and tutors $(\mathrm{T})$. A design process is either formulated by a single actor that is the tutor or the student, or by both, when the first issue is enacted by the tutor and the second by the student or inversely. We distinguish four types of interactions: $\mathrm{S}>\mathrm{S}$ or student to student; $\mathrm{S}>\mathrm{T}$ or student to tutor; $\mathrm{T}>\mathrm{S}$ or tutor to student and $\mathrm{T}>\mathrm{T}$ or tutor to tutor.

The segmentation and coding of a crit session results in a transformation of the protocol video into a sequence of codes. Where the content of a segment does not match one of the six FBS codes it is coded as $\mathrm{O}$ (other). Typical segments coded as $\mathrm{O}$ include jokes and social interactions. Similarly, when a transition does not match one of the eight FBS processes it is coded as O (other). Standard statistical measures are used to describe a coded session.

\subsection{P-S index and moving P-S indicator}

A meta-cognitive understanding of a design session can be gained by measuring the P-S index. It gives an indication of the focus of the design activity (Jiang et al., 2014). It is expressed as the ratio of the sum of problem related issues ( $\mathrm{R}, \mathrm{F}$ and $\mathrm{Be}$ ) to the sum of solution related issue ( $\mathrm{S}$ and $\mathrm{Bs}$ ). If the $\mathrm{P}-\mathrm{S}$ index has a value between 0 and 1, it means that the design activity is solution-focused. If the P-S index value is over 1, the focus is mainly on the problem. We were looking for a symmetrical representation of the focus of the design activity, so we used a refined version of the P-S index, the P-S indicator, whose value ranges between -1 and 1 . A moving P-S indicator illustrates the variation of the problemsolution focus temporally across the session. A moving window of $\mathrm{n}$ segments defines the size of the local indicator. The indicator is locally expressed by the subtraction of the sum of solution related issue ( $\mathrm{S}$ and $\mathrm{Bs}$ ) from the sum of problem related issue ( $\mathrm{R}, \mathrm{F}$ and $\mathrm{Be}$ ), divided by $\mathrm{n}$. That way, the moving $\mathrm{P}-$ $\mathrm{S}$ indicator has a value between -1 and 1 . When its value is 0 , it implies that the local design activity is equally distributed between the problem and solution spaces. If the value is between 0 and 1 , the design activity is problem-focused and if the value is between 0 and -1, the design activity is solution focused.

\section{Data description}

For this case study a single team of two students of the design studio Architecture \& Construction at Ghent University was observed and recorded at four different critique sessions during the design studio, labelled sessions 1 through 4 . The number of tutors varies between the sessions, from 1 to 3 . The design brief consisted of the refurbishment of three high-rise apartment buildings into a co-housing complex and a parking area. The first and the second session were recorded on the fourth and fifth weeks of the studio which was 14 weeks long. The last two sessions were recorded during the two last weeks of the studio. The last session was the final presentation of the project. The lengths of the sessions varied from 45 minutes to more than 2 hours. The longest was session 3 , that took place before the final presentation. The level of detail of the project made it necessarily longer, in order for tutors to cover the entire design. All the results are normalized to remove the length of the session as a variable. A description of the design brief and content of each session is presented in Pauwels et al. (2015).

\section{Results}

Each session was transcribed and coded using the two schemes presented above. Tutors and students are considered as a single entity, labelled by actor type. We aim to capture the design activity during the design critique, by considering the navigation of the problem-solution design space. We explore the cognitive design behavior of each actor, by taking into account their interactions.

\subsection{General distributions of design process and interactions}

The distributions of design processes for a single session give a description of the nature of the design activity (Kan and Gero, 2017). The normalized distributions of the eight processes of the FBS ontology are described in Table 1. Analysis, Evaluation, and Reformulation 1 dominate the distributions of processes for every session. Synthesis and Reformulation 2 occurred fewer times but also have a similar distribution for all three sessions. The distribution of Evaluation process drops for session 4: it reaches 
only $11.1 \%$, whereas its distribution for session 1 , session 2 and session 3 is respectively $32.2 \%, 28.9 \%$ and $29.8 \%$. Comparatively, the distribution of Analysis process in session 4 is almost twice as its distribution for session 1 . The distributions for session 2 and 3 are similar $(30.4 \%$ and $33.6 \%)$. The normalized occurrences of Reformulation 1 are stable across the session, as its mean is $24.5 \%$ with a relatively low standard deviation (3.7).

Table 1. Normalized distribution of FBS design processes for each session (\%)

\begin{tabular}{r|ccccc} 
& Session 1 & Session 2 & Session 3 & Session 4 & Mean (SD) \\
\hline Formulation & 4.2 & 1.5 & 1.4 & 0.0 & $1.8(1.8)$ \\
Synthesis & 7.7 & 9.9 & 7.0 & 2.8 & $6.9(3.0)$ \\
Analysis & 26.6 & 30.4 & 33.6 & 50.0 & $35.1(10.3)$ \\
Evaluation & 32.1 & 28.9 & 29.8 & 11.1 & $25.5(9.7)$ \\
Documentation & 0.00 & 1.5 & 0.7 & 2.8 & $1.3(1.2)$ \\
Reformulation 1 & 25.2 & 19.8 & 24.2 & 28.7 & $24.5(3.7)$ \\
Reformulation 2 & 4.2 & 5.7 & 3.1 & 3.7 & $4.2(1.1)$ \\
Reformulation 3 & 0.0 & 2.3 & 0.2 & 0.9 & $0.9(1.0)$
\end{tabular}

The distributions for the four interactions associated with one of the eight FBS design process show variations across the four sessions (Table 2).

Table 2. Normalized distribution of FBS design process for each interaction (\%)

\begin{tabular}{r|ccccc}
\multicolumn{1}{c}{} & Session 1 & Session 2 & Session 3 & Session 4 & Mean (SD) \\
\hline Student to student $(S>S)$ & 29.4 & 15.6 & 6.8 & 7.4 & $14.8(10.5)$ \\
Student to tutor $(S>T)$ & 20.9 & 20.2 & 28.2 & 21.3 & $22.7(3.7)$ \\
Tutor to student $(T>S)$ & 13.3 & 15.6 & 19.0 & 12.0 & $15.0(3.1)$ \\
Tutor to tutor $(T>T)$ & 36.4 & 48.7 & 46.0 & 59.3 & $47.6(9.4)$
\end{tabular}

Design processes of students alone ( $\mathrm{S}>\mathrm{S}$ interactions) decrease over the four sessions. In session 1, it represents $29.4 \%$ of the overall design process, and drops to $7.4 \%$ for session 4 . Design processes of tutors ( $\mathrm{T}>\mathrm{T}$ interactions) are always the most dominant. In session $4, \mathrm{~T}>\mathrm{T}$ interaction takes over the critique $(59.3 \%)$. Design processes that occurred during a tutor to student $(\mathrm{T}>\mathrm{S})$ or student to tutor interaction $(\mathrm{S}>\mathrm{T})$ show less variations across the sessions than the other two types of interactions. The proportion of $\mathrm{S}>\mathrm{T}$ design process is always greater than the proportion of $\mathrm{T}>\mathrm{S}$ design process. In session 3 , the occurrences of $\mathrm{S}>\mathrm{T}(28.2 \%)$ and $\mathrm{T}>\mathrm{S}(19.0 \%)$ design process is relatively higher than for the other sessions ( $\mathrm{S}>\mathrm{T}$ between $20 \%$ and $22 \%, \mathrm{~T}>\mathrm{S}$ between $12 \%$ and $16 \%$ ). The actor's participation in the critique, in terms of design activity, changed across the session. Design processes formulated by students tend to decrease whereas design processes expressed by tutors tend to increase. The distributions of $\mathrm{S}>\mathrm{T}$ and $\mathrm{T}>\mathrm{S}$ design process vary only slightly except in session 3 .

\subsection{Navigation of the problem-solution space}

In order to analyze the situation of the design activity in terms of the design space, we measure the P-S indicator for each session (Table 3). All of the P-S indicator values are less than 0 which indicate that the overall design activity is solution-focused. The decrease of the P-S indicator from session to session denotes that more and more attention is given to the design solution.

Table 3. P-S indicator for each session

\begin{tabular}{c|cccc} 
& Session 1 & Session 2 & Session 3 & Session 4 \\
\hline$P-S$ indicator & -0.28 & -0.48 & -0.58 & -0.86
\end{tabular}

To gain further insight into the navigation of the design space during the session, we plotted the moving P-S indicator for each session (Figure 2). A moving window of 1/10 of the length of the session was 
chosen. Here, we present a qualitative analysis of the results in Figure 2. During session 1, the first three quarters of the session exhibits a near equal distribution of design effort between the problem and solution spaces, with a slight tendency to focus on the solution (Figure 2a). During session 2, for the first three quarters of the session, the P-S indicator stays around -0.5 which indicates that the focus is mainly on the solution (Figure 2b). The increase around segment 430 implies that more verbalizations occurred concerning the problem space. During session 3, there is a gradual decrease of the moving P-S indicator (Figure 2c). A similar increase to that in session 2 appears at the beginning of the session around segment 180. Session 4 explicitly focuses on the solution, as the P-S indicator value varies between -1.0 and -0.6 .

(a)

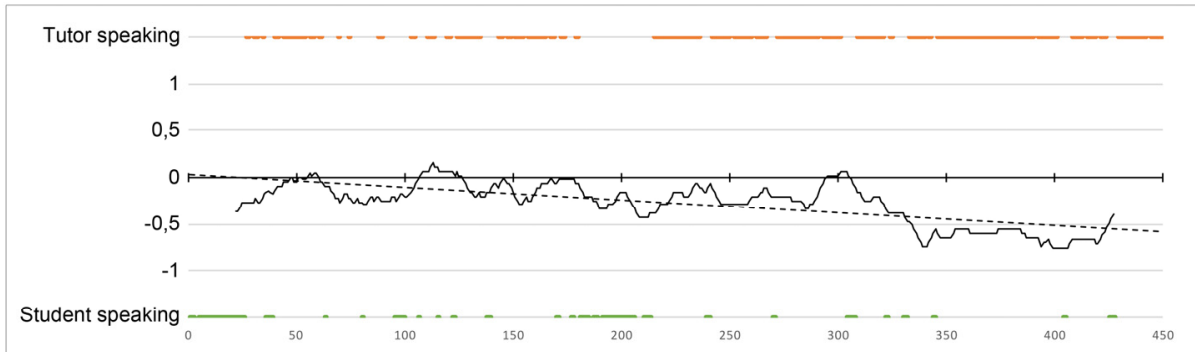

(b)

Tutor speaking

1

0,5

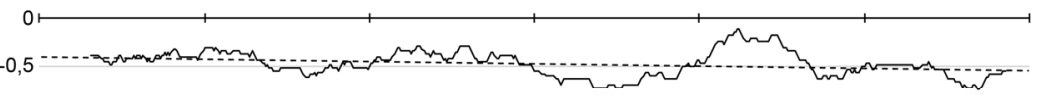

$-1$

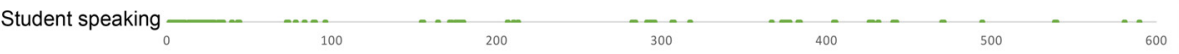

Tutor speaking

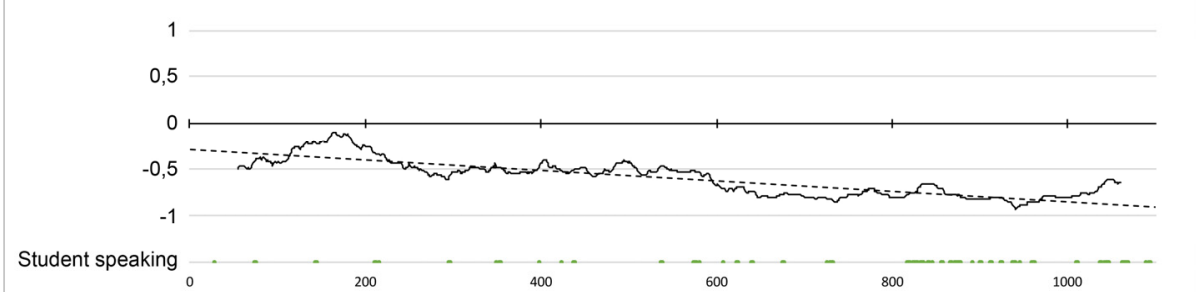

(c)

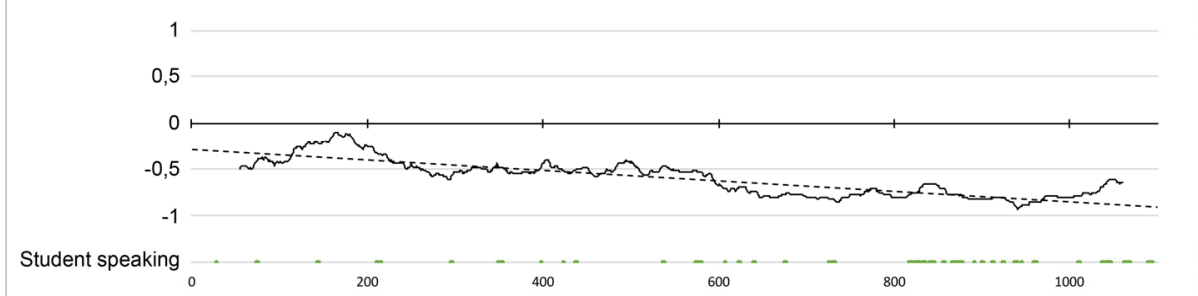

)

Tutor speaking

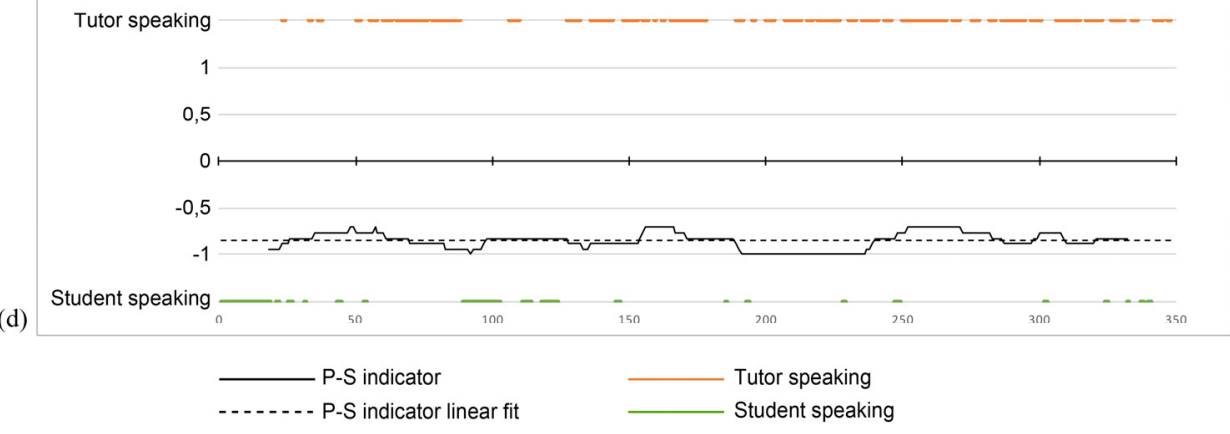

Figure 2. Moving Problem - Solution indicator for the design activity: (a) Session 1, (b) Session 2, (c) Session 3, (d) Session 4

\subsection{First order Markov models}

First order Markov models express the probability of transitioning from a given state to another state. The purpose of using this tool to analyze each protocol is to reveal design patterns specific to a data set (Kan and Gero, 2010; Yu and Gero, 2016). The Markov analysis produces a probability matrix based 
on the sequence of event states in the data set. In our data, 12 states are considered, that are related to design issues (one of the 6 design issues from the FBS ontology) and speakers (students or tutors). The transition probability varies between 0 and 1 . Transitions with a high probability (above the selected threshold of 0.25 , three times the random probability) are representative of the dominant design issues transitions for each session (Figure 3). The transition matrix shows the probability of any transition, including the eight theoretical design processes described by the FBS ontology.

(a)

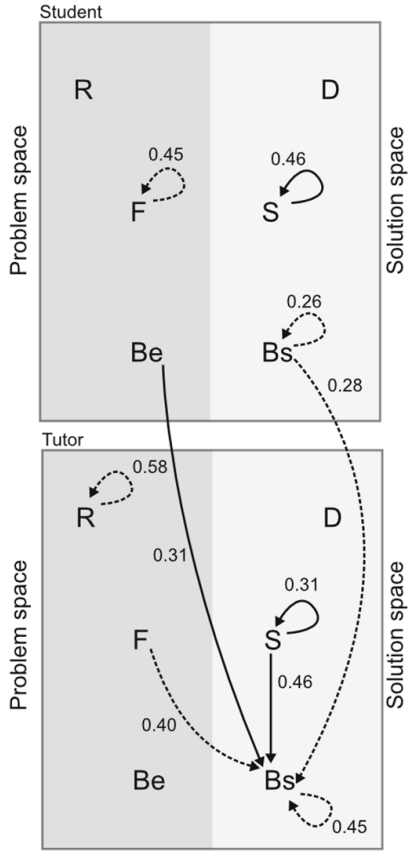

(c)

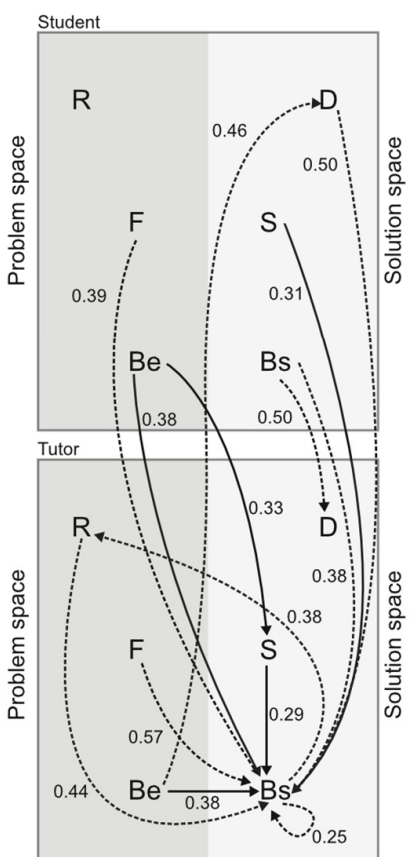

(b)
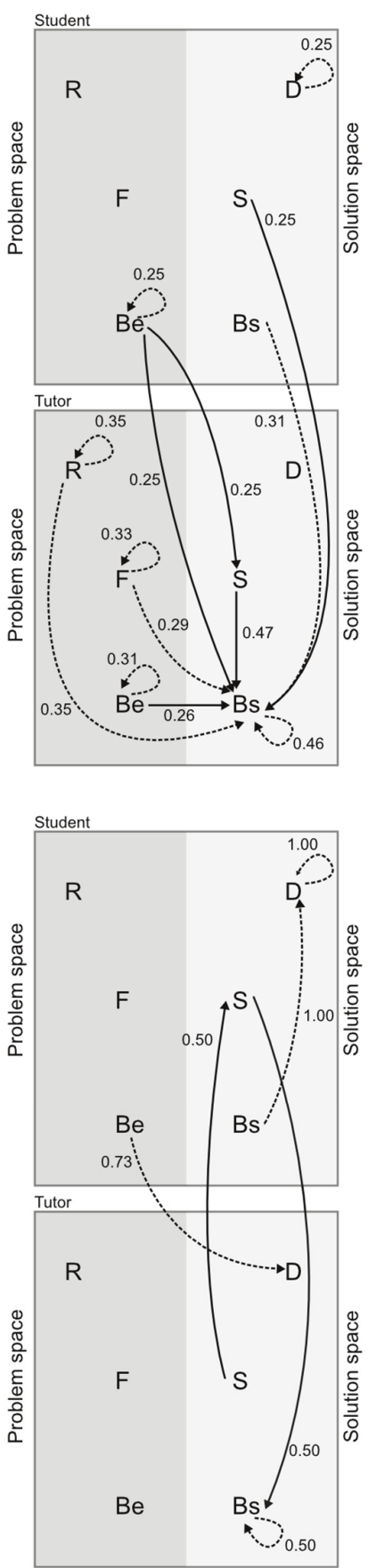

(d)

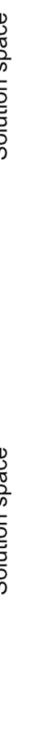

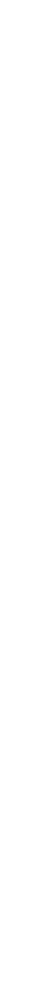

Figure 3. Representation of prevalent design issue transitions based on their probability: (a) Session 1, (b) Session 2, (c) Session 3, (d) Session 4 
In the first session, three design processes have a high probability: Reformulation $1 \mathrm{~S}>\mathrm{S}$ and $\mathrm{T}>\mathrm{T}$, Evaluation (from Be to Bs) $\mathrm{S}>\mathrm{T}$ and Analysis $\mathrm{T}>\mathrm{T}$ (Figure 3(a)). Most of the transitions are in the same space and expressed by a single actor. In the session 2, $\mathrm{T}>\mathrm{T}$ Analysis and Evaluation (from $\mathrm{Be}$ to $\mathrm{Bs}$ ), as well $\mathrm{S}>\mathrm{T}$ Synthesis, Analysis and Evaluation (from Be to Bs) are above the probability threshold (Figure 3(b)). More transitions from students to tutors, as well as from the problem space to the solution space are prevalent in the Markov analysis for that session. In session 3, the same design processes are above the threshold: Synthesis $(\mathrm{S}>\mathrm{T})$, Analysis $(\mathrm{S}>\mathrm{T}$ and $\mathrm{T}>\mathrm{T})$ and Evaluation from $\mathrm{Be}$ to $\mathrm{Bs}(\mathrm{S}>\mathrm{T}$ and $\mathrm{T}>\mathrm{T}$ ) (Figure 3(c)). A high variety of transitions above the threshold appears in the model for that session, which encompasses the highest dynamism in the transitions from both spaces and actors. In session 4, the two design processes with a high probability are $\mathrm{S}>\mathrm{T}$ Analysis, and $\mathrm{T}>\mathrm{S}$ Reformulation 1. There is a limited number of transitions above the threshold (only 4), and most of the transitions sit in the solution space.

The Markov model offers a quantitative probabilistic description of the design transitions for each session, that we can explore to capture qualitative information about each session. Most of the highest probability design process are expressed by a transition from the problem space to the solution space (Synthesis, Evaluation Be to Bs), or are set in the solution space only (Analysis, Reformulation 1). Some similarities can be pointed out across the session: Analysis (either $\mathrm{T}>\mathrm{T}$ or $\mathrm{S}>\mathrm{T}$ ) always has a high probability, as does Evaluation (Be to Bs), except for session 4. Differences are found in the probability of Reformulation 1 that is above the threshold only for session 1 (both $\mathrm{T}>\mathrm{T}$ and $\mathrm{S}>\mathrm{S}$ ) and for session 4 $(\mathrm{T}>\mathrm{S})$. Also, the Synthesis process has a higher probability only in session 2 and session 3 . When considering the transitions between design spaces and between actors, we can discern a gradual change across sessions 1 to 3 . In session 1, most transitions are in the same space, expressed by the same actor. In session 2, the same kind of transitions are found, but the number of transitions between spaces and between actors increases. Session 3 shows the most diversity in the type of transitions, in the navigation from one space to another, and from one actor to another. In that session only, a transition from the solution space to the design space appears with a high probability. Apart from $\mathrm{S}>\mathrm{T}$ Analysis, the other transitions from session 4 are only found in that session.

\subsection{Correspondence analysis between design process and interactions}

A correspondence analysis between the types of interaction and the design processes provides a qualitative understanding of the design behavior for the four sessions. The five prevalent design processes for the sessions were selected for the correspondence analysis: Synthesis, Analysis, Evaluation (from Bs to Be), Evaluation (from Be to Bs) and Reformulation 1. The FactoMiner package in RStudio was used for the analysis (Lê et al., 2008). A two-dimension graph was plotted to represent the results for each session (Figure 4).

The results of the correspondence analysis for session 1, plotted in a two-dimension graph (Figure 4(a)) cover $98.9 \%$ of the data variance (Dim $1=66.4 \%$ and Dim $2=32.5 \%$ ). S $>$ S appears on the opposite side of dimension 1 to the other three interactions. The results from the correspondence analysis imply that $\mathrm{S}>\mathrm{S}$ is strongly related with Reformulation $1 . \mathrm{T}>\mathrm{T}$ and $\mathrm{S}>\mathrm{T}$ are in the same quadrant with the Analysis and Evaluation ( $\mathrm{Bs}>\mathrm{Be}) . \mathrm{T}>\mathrm{S}$ is in another quadrant, with the Evaluation (Be $>\mathrm{Bs}$ ). The results of the correspondence analysis for session 2, plotted in a two-dimension graph (Figure 4(b)) cover 92.6 $\%$ of the data variance (Dim $1=56.5 \%$ and Dim $2=36.1 \%$ ). S $>$ S appears on the opposite side of dimension 1 than $\mathrm{T}>\mathrm{T}$ and $\mathrm{T}>\mathrm{S}$, similarly to session 2 . It is again closely related to Reformulation 1 process. $\mathrm{T}>\mathrm{T}$ sits by itself in a quadrant with the two types of Evaluation. $\mathrm{T}>\mathrm{S}$ appears to be related to Synthesis, and $\mathrm{S}>\mathrm{T}$ to Analysis.

The results of the correspondence analysis for session 3, plotted in the a-dimension graph (Figure 4(c)) cover $92.4 \%$ of the data variance (Dim $1=34.7 \%$ and $\operatorname{Dim} 2=57.7 \%$ ). For that session, all the interactions appear on different quadrants. $\mathrm{S}>\mathrm{S}$ is in the same quadrant as Reformulation $1 . \mathrm{T}>\mathrm{T}$ is situated close to Evaluation ( $\mathrm{Bs}>\mathrm{Be})$. As well as for session 2, $\mathrm{T}>\mathrm{S}$ seems to be related to Synthesis, and $\mathrm{S}>\mathrm{T}$ to Analysis.

The results of the correspondence analysis for session 4, plotted in a two-dimension graph (Figure 4(d)) cover $95.5 \%$ of the data variance ( $\operatorname{Dim} 1=37.1 \%$ and $\operatorname{Dim} 2=58.4 \%$ ). For that session, $\mathrm{S}>\mathrm{S}$ and $\mathrm{T}>\mathrm{T}$ are in the same quadrant, with Reformulation 1. $\mathrm{T}>\mathrm{S}$ appears on the same side of dimension 1 than $\mathrm{T}>\mathrm{T}$ 
and $\mathrm{S}>\mathrm{S}$ but in the upper quadrant. $\mathrm{S}>\mathrm{T}$ sits by itself on the left side of dimension 1, in the same quadrant as Analysis and Evaluation $\mathrm{Bs}>\mathrm{Be}$.

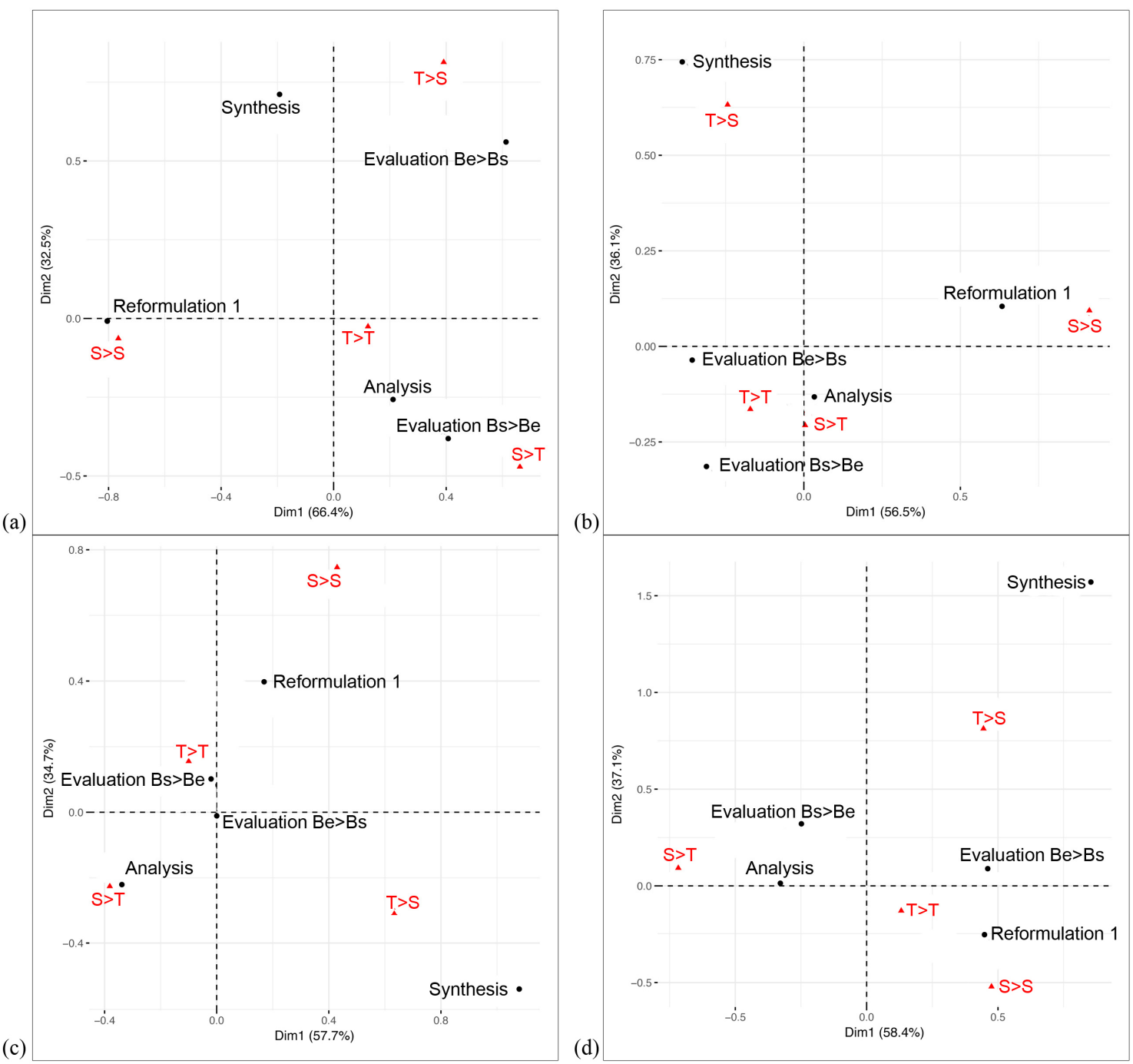

Figure 4. Correspondence analysis of interactions and design process for (a) session 1, (b) session 2, (c) for session 3 and (d) session 4. The dimensions (Dim1 and Dim2) across the graphs are not commensurate

The correspondence analysis results represent the differences between each interactions depending on their relationship with the prevalent process. $\mathrm{S}>\mathrm{S}$ is always related to Reformulation 1, and is the only interaction that shows little variation across the sessions. For the first three sessions, $\mathrm{S}>\mathrm{S}$ interactions is by itself in a quadrant. $\mathrm{T}>\mathrm{T}$ sits in the same quadrant as Evaluation $(\mathrm{Bs}>\mathrm{Be})$ for the first three sessions. The cognitive behavior linked with $\mathrm{T}>\mathrm{T}$ interactions has more variations across the sessions. The $\mathrm{T}>\mathrm{S}$ interaction is related to Synthesis for 3 sessions and Evaluation ( $\mathrm{Be}>\mathrm{Bs}$ ) for two sessions. $\mathrm{S}>\mathrm{T}$ is always in the same quadrant as Analysis, and related to Evaluation $(\mathrm{Bs}>\mathrm{Be}$ ) for two sessions.

\section{Discussion}

This case study illustrates how the design activity evolves throughout the semester for a single student team's crit sessions. It reveals the differences and similarities between the four sessions studied. Students' participation in the formulation of design process decreases across the session and is always lower than the tutors' generation of design processes. This aligns with Schön's (1985) description of 
students during the critiques, as learners by imitation and observation. The distribution of the eight processes (Formulation, Synthesis, Analysis, Evaluation, Documentation, Reformulation 1, Reformulation 2 and Reformulation 3) is quite similar for each session except for session 4 which was the final jury. Nevertheless, when we looked at the navigation of the design space (P-S indicator), we could see clear differences in the focus of the design activity for each session. The focus of the critique sessions is mainly on the solution which coincides with the solution-focused tendency of architectural design (Kan and Gero, 2011). The change of the P-S indicator across the sessions maps onto the overall timing of the design project. Within each session, the moving P-S indicator fluctuates, which captures the evolution of the transitions between the problem and solution space, as has been described in previous empirical studies on architectural design (Yu et al., 2015).

The first order Markov model showed the probability of Analysis and Evaluation (Be to Bs) either from $\mathrm{T}>\mathrm{T}$ or $\mathrm{S}>\mathrm{T}$ is high for most sessions. Both those design processes are expressed by a transition between a design issue in the problem space to a design issue in the solution space. The other two design processes with a high probability were Synthesis (for session 2 and 3) and Reformulation 1 (for session 1 and 4). Synthesis is constructed by a transition from a design issue in the problem space to a design issue in the solution space. Reformulation 1 is a transition from a structure issue (S) to another structure issue (S), therefore, takes place in the solution space. The Markov analysis shows that the direction in which the design space is navigated is primarily from the problem space to the solution space.

The results from the correspondence analysis provide further qualitative insights into the cognitive design behavior of each actor along the sessions. The participation and interaction of the actors vary across the sessions as well as the type of design process associated with each interaction. Changes observed might be due to the general evolution of the design activity, and to the project timing. Nevertheless, we could observe tendencies linked with each interaction. Students by themselves verbalize design issues in the solution space (Reformulation 1). While interacting with tutors, they also explore the solution space (Analysis) and navigate from the solution space to the problem space and inversely (Synthesis, Evaluation Be $>\mathrm{Bs}$, Evaluation $\mathrm{Bs}>\mathrm{Be}$ ). Tutors verbalize utterances in both spaces, and seem to have more capacity to navigate the design space. Although, the design activity becomes more and more focused on the solution space, the tutors drove the design activity towards the problem space for the first three critique sessions as Evaluation $\mathrm{Bs}>\mathrm{Be}$ is related to $\mathrm{T}>\mathrm{T}$ and $\mathrm{S}>\mathrm{T}$ interaction. Each actor influences the navigation of the design space in a different way. The description of tutors' and students' participation in the design critiques in terms of design process illustrates the position they took during the critiques: students present and defend their design solution while tutors analyze and question the design situation.

The aim of the design critique is both to evaluate and guide students in continuing their design. The coevolution of the design space is a characteristic of the design activity and implies a navigation from one to the other (Maher and Poon, 1996; Dorst and Cross, 2001). Novices and experts navigate the problemsolution spaces in different ways depending on their knowledge of designing (Cross, 1982; Dorst and Cross, 2001). Design novices tend to be fixated on a solution early in the design process and that can hinder design potentials (Lawson and Dorst, 2009). Those statements apply to our observations of the four design critiques. In the context of the design studio, the challenge is for students to learn how to reflect in creative designerly ways (Cross, 1982). Therefore, shifting from the solution space to the problem space is needed to enrich the design process and design outcome. That role, although not that dominant, is taken by the tutor in the critique sessions that where presented in the paper.

\section{Conclusion and future work}

This exploratory case study served as a test bed to formulate future hypotheses to be tested in our further work. In this single case study, we found that: (1) over the semester, $S>S$ interactions tend to decrease while $\mathrm{T}>\mathrm{T}$ interactions increase, (2) the design activity becomes more solution focused across the sessions, (3) the probability of design processes constructed by a transition from a design issue in the problem space to a design issue in the solution space is dominant and (4) occurrences of design processes constructed by a transition from the solution space to the problem space appear mainly during $\mathrm{T}>\mathrm{T}$ or $\mathrm{S}>\mathrm{T}$ interactions. Critiques sessions are part of the studio pedagogy. The effects of that pedagogy are observed and evaluated through students' design, the outcome of their design activity, but not the activity itself. The 
objective of our future work will be to capture the critique sessions as well as design sessions in between, to provide a better understanding of the critique's pedagogy and its effects. We intend to refine and test hypotheses by analyzing multiple cases in order to have a statistically reliable sample to generalize our future findings. This paper presented different methods and tools, used for the case study, and that will be applied as part of our methodology. The articulation between the evaluation of the navigation of the design space, the evolution of cognitive design behavior and the role of the tutor/student in terms of design education pedagogy through empirical research will provide a new empirical basis to investigate the studio pedagogy and construct a better understanding of how students learn how to design.

\section{Acknowledgements}

This research is funded in part by a grant from the US National Science Foundation to the second author, Grant No. EEC-1463873.

\section{References}

Adams, R.S. and Siddiqui, J.A. (2016), Analyzing design review conversations, Purdue University Press, West Lafayette, Indiana.

Adams, R.S., Cardella, M. and Purzer, Ş. (2016a), “Special Issue: Design Review Conversations”, Design Studies, Vol. 45, pp. 1-158.

Adams, R.S., Cardella, M. and Purzer, Ş. (2016b), "Analyzing design review conversations: Connecting design knowing, being and coaching", Design Studies, Vol. 45, pp. 1-8. https://doi.org/10.1016/j.destud.2016.03.001

Adams, R.S., McMullen, S. and Fosmire, M. (2016c), “Co-Designing Review Conversations”, CoDesign, Vol. 12 No. 1-2, pp. 1-131.

Anthony, K.H. (1991), Design juries on trial: the renaissance of the design studio, Van Nostrand Reinhold, Prentice-Hall.

Cardoso, C., Badke-Schaub, P. and Eris, O. (2016), "Inflection moments in design discourse: How questions drive problem framing during idea generation", Design Studies, Vol. 46, pp. 59-78. https://doi.org/10.1016/j.destud.2016.07.002

Cross, N. (1982), "Designerly ways of knowing", Design Studies, Vol. 3, pp. 221-227.

Dinham, S.M. (1989), "Teaching as design: theory, research and implications for design teaching", Design Studies, Vol. 10 , pp. 80-88.

Dong, A., Garbuio, M. and Lovallo, D. (2016), “Generative sensing in design evaluation”, Design Studies, Vol. 45, pp. 68-91. https://doi.org/10.1016/j.destud.2016.01.003

Dorst, K. and Cross, N. (2001), "Creativity in the design process: co-evolution of problem-solution", Design Studies, Vol. 22, pp. 425-437.

Dorta, T., Kinayoglu, G. and Boudhraâ, S. (2016), "A new representational ecosystem for design teaching in the studio", Design Studies, Vol. 47, pp. 164-186. https://doi.org/10.1016/j.destud.2016.09.003

Dutton, T.A. (1987), “Design and Studio Pedagogy”, Journal of Architural Education, Vol. 41, No. 1, pp. 16-25. https://doi.org/10.2307/1424904

Ericsson, K.A. and Simon, A.H. (1984), Protocol Analysis: Verbal reports as data, MIT Press.

Ferreira, J., Christiaans, H. and Almendra, R. (2016), "A visual tool for analysing teacher and student interactions in a design studio setting", CoDesign, Vol. 12, pp. 112-131. https://doi.org/10.1080/15710882.2015.1135246

Gero, J.S. (1990), "Design prototypes: a knowledge representation schema for design", AI Magazine, Vol. 11 No. 4, pp. 26-36. https://doi.org/10.1609/aimag.v11i4.854

Gero, J.S. and Jiang, H. (2016), "Exploring the design cognition of concept design reviews using the FBS-Based protocol analysis", In: Adams, R.S. and Siddiqui, J.A. (Eds.), Analyzing Design Review Conversations, Purdue University Press, West Lafayette, Indiana.

Gero, J.S. and Kannengiesser, U. (2007), "A function-behavior-structure ontology of processes", Artificial Intelligence for Engineering Design, Analysis and Manufacturing, Vol. 21 No. 4, pp. 379-391. https://doi.org/10.1017/S0890060407000340

Gero, J.S. and Mc Neill, T. (1998), "An approach to the analysis of design protocols", Design Studies, Vol. 19, pp. 21-61.

Goldschmidt, G. (2002), “'One-on-One’: A Pedagogic Base for Design Instruction in the Studio”, Proceedings of "Common Ground", Design Research Society International Conference, Brunel University, Stoke-on-Trent, September 5-7, 2002, Staffordshire University Press, pp. 430-437.

Goldschmidt, G., Hochman, H. and Dafni, I. (2010), “The design studio "crit": Teacher-student communication", Artificial Intelligence for Engineering Design Analysis and Manufacturing, Vol. 24 No. 3, pp. 285-302. https://doi.org/10.1017/S089006041000020X 
Jiang, H., Gero, J.S. and Yen, C.-C. (2014), "Exploring designing styles using a problem-solution division", Design Computing and Cognition'12, Springer, pp. 79-94.

Kan, J.W. and Gero, J.S. (2010), "Exploring quantitative methods to study design behavior in collaborative virtual workspaces", New Frontiers, Proceedings of the 15th International Conference on CAADRIA.

Kan, J.W. and Gero, J.S. (2011), "Comparing designing across different domains: An exploratory case study", 18th International Conference on Engineering Design (ICED’11), Copenhagen, Denmark.

Kan, J.W. and Gero, J.S. (2017), Quantitative Methods for Studying Design protocols, Springer.

Khaidzir, K.A.M. and Lawson, B. (2013), "The cognitive construct of design conversation", Research in Engineering Design. Vol. 24 No. 4, pp. 331-347. https://doi.org/10.1007/s00163-012-0147-8

Lawson, B. (2006), How designers think: the design process demystified, 4th. ed., Elsevier/Architectural Press, Amsterdam.

Lawson, B. and Dorst, K. (2009), Design Expertise, Architectural Press, Routledge.

Lê, S., Josse, J. and Husson, F. (2008), "FactoMineR: An R Package for Multivariate Analysis", Journal of Statistical Software, Vol. 25 No. 1, pp. 1-18.

Maher, M.L. and Poon, J. (1996), "Modelling design exploration as co-evolution", Microcomputers in Civil Engineering, Vol. 11, pp. 195-210.

Mewburn, I. (2012), "Lost in translation: Reconsidering reflective practice and design studio pedagogy", Arts and Humanities in Higher Education, Vol. 11 No. 4, pp. 363-379. https://doi.org/10.1177/1474022210393912

Oak, A. and Lloyd, P. (2016), “'Throw one out that's problematic': performing authority and affiliation in design education", CoDesign, Vol. 12, pp. 55-72.

Oh, Y., Ishizaki, S., Gross, M.D. and Yi-Luen Do, E. (2013), "A theoretical framework of design critiquing in architecture studios", Design Studies, Vol. 34 No. 3, pp. 302-325. https://doi.org/10.1016/j.destud.2012.08.004

Pauwels, P., Strobbe, T. and De Meyer, R. (2015), "Analysing how constraints impact architectural decisionmaking”, International Journal of Design Science and Technology, Vol. 21 No. 1, pp. 83-111.

Schön, D. (1985), The Design Studio, RIBA, London.

Schön, D. (1988), "Designing: rules, types and worlds", Design Studies, Vol. 9 No. 3, pp. 181-190. https://doi.org/10.1016/0142-694X(88)90047-6

Visser, W. (2006), The cognitive artefact of designing, Lawrence Erlbaum Associates.

Wolmarans, N. (2016), "Inferential reasoning in design: Relations between material product and specialised disciplinary knowledge”, Design Studies, Vol. 45, pp. 92-115. https://doi.org/10.1016/j.destud.2015.12.003

Yu, R. and Gero, J.S. (2016), "An empirical basis for the use of design patterns by architects in parametric design", International Journal of Architectural Computing, Vol. 14 No. 3, pp. $289-302$. https://doi.org/10.1177/1478077116663351

Yu, R., Gu, N., Ostwald, M. and Gero, J.S. (2015), "Empirical support for problem-solution co-evolution in a parametric design environment", Artificial Intelligence for Engineering Design, Analysis and Manufacturing, Vol. 25 No. 1, pp. 33-44. https://doi.org/10.1017/S0890060414000316

Julie Milovanovic, PhD Candidate

UMR AUU-CRENAU, Architecture

6 quai Francois Mitterand, 44200 Nantes, France

Email: julie.milovanovic@crenau.archi.fr 